International Journal of Pure and Applied Mathematics

Volume 88 No. 4 2013, 475-482

ISSN: 1311-8080 (printed version); ISSN: 1314-3395 (on-line version)

url: http://www.ijpam.eu

doi: http://dx.doi.org/10.12732/ijpam.v88i4.3

\title{
GEOMETRICAL HYPERCOMPLEX COUPLING BETWEEN ELECTRIC AND GRAVITATIONAL FIELDS
}

\author{
J.A.P.F. Marão ${ }^{1}$, M.F. Borges ${ }^{2} \S$ \\ ${ }^{1}$ Department of Mathematics \\ Federal University of Maranhão - São Luís-MA \\ 65085-580, Maranhão, BRAZIL \\ ${ }^{2}$ UNESP - São Paulo State University \\ S.J. Rio Preto Campus \\ 15054-000, São José do Rio Preto, BRAZIL
}

\begin{abstract}
The present work shows a coupling of electrical and gravitational fields through Cauchy-Riemann conditions for quaternions present in a previous paper [1]. It is also obtained an extended version of the Laplace-like equations for quaternions, now written in terms of both electric and gravitational fields.
\end{abstract}

AMS Subject Classification: 30G99, 30E99

Key Words: quaternions, Laplace's equations, quate

\section{Initial Provisions}

Throughout this work, are considered quaternionic functions which follow the pattern $f_{i}(t, x, y, z)$, with $i=1,2,3,4$, where $t$ is the time and the coordinates $x, y$ and $z$ are considered the spatial coordinates. Thus, the quaternion $\mathrm{q}$ is written here as follows;

$$
q=t+x i+y j+z k
$$

or

$$
q=t+\vec{u} .
$$

The next section based on a paper by Borges and Machado [2] shows a set

Received: May 7, 2013

(C) 2013 Academic Publications, Ltd.

$\S$ Correspondence author url: www.acadpubl.eu 
Cauchy-Riemann like relations for quaternionic functions. These equations will be adapted to the particular case where $x_{1}$ will be replaced by the time and the other coordinates $x_{2}, x_{3}$ and $x_{4}$ will be identified here for $x, y$ and $z$, respectively.

\section{Cauchy-Riemann Conditions for Quaternionic Functions}

The conditions named here as Cauchy-Riemann like relations for quaternionic functions, are treated in detail in [1]. It follows the theorem:

Theorem 1. For any pair pontis $a$ and $b$ and any path joining them simply conect subdomain of the four-dimmensional space, the integral $\int_{a}^{b} f d q$ is independent form the given path if and only if there is a function $F=$ $F_{1}+F_{2} i+F_{3} j+F_{4} k$ such that $\int_{a}^{b} f d q=F(a) F(b)$, and satisfying the following relations:

$$
\begin{aligned}
\frac{\partial F}{\partial t} & =\frac{\partial F_{2}}{\partial x}=\frac{\partial F_{3}}{\partial y}=\frac{\partial F_{4}}{\partial z}, \\
\frac{\partial F_{2}}{\partial t} & =-\frac{\partial F_{1}}{\partial x}=-\frac{\partial F_{3}}{\partial z}=\frac{\partial F_{4}}{\partial y}, \\
\frac{\partial F_{3}}{\partial t} & =-\frac{\partial F_{1}}{\partial y}=-\frac{\partial F_{2}}{\partial z}=\frac{\partial F_{4}}{\partial x}, \\
\frac{\partial F_{4}}{\partial t} & =\frac{\partial F_{1}}{\partial z}=-\frac{\partial F_{2}}{\partial y}=\frac{\partial F_{3}}{\partial x} .
\end{aligned}
$$

Proof. The proof of this theorem can be analyzed in greater detail in [1].

\section{The Laplace's Equations}

In this section it will be determined that from the relations showed in Theorem 1 , naturally follows a new set of quaternionic Laplacelike equations. Therefore, the functions that make up the quaternionic function depend on $t, x, y$ and $z$ and are supposed of class $C^{2}$.

The first step to obtain the Laplace equations is the derivation of equations (5), (6), (7) and (8) over $t, x, y$ and $z$. That will be done as follows: Deriving 
the conditions of equation (5), we have that:

$$
\begin{aligned}
& \frac{\partial^{2} F_{1}}{\partial y \partial t}=\frac{\partial^{2} F_{2}}{\partial t \partial x}=\frac{\partial^{2} F_{3}}{\partial t \partial y}=\frac{\partial^{2} F_{4}}{\partial t \partial z} \\
& \frac{\partial^{2} F_{1}}{\partial t \partial x}=\frac{\partial^{2} F_{2}}{\partial x^{2}}=\frac{\partial^{2} F_{3}}{\partial x \partial y}=\frac{\partial^{2} F_{4}}{\partial x \partial z} \\
& \frac{\partial^{2} F_{1}}{\partial y \partial t}=\frac{\partial^{2} F_{2}}{\partial y \partial x}=\frac{\partial^{2} F_{3}}{\partial y^{2}}=\frac{\partial^{2} F_{4}}{\partial z \partial y} \\
& \frac{\partial^{2} F_{2}}{\partial t \partial z}=\frac{\partial^{2} F_{2}}{\partial z \partial x}=\frac{\partial^{2} F_{3}}{\partial z \partial y}=\frac{\partial^{2} F_{4}}{\partial z^{2}} .
\end{aligned}
$$

Deriving the conditions of equation (6), we obtain:

$$
\begin{aligned}
& \frac{\partial^{2} F_{2}}{\partial t^{2}}=-\frac{\partial^{2} F_{1}}{\partial t \partial x}=-\frac{\partial^{2} F_{3}}{\partial t \partial z}=\frac{\partial^{2} F_{4}}{\partial t \partial y} \\
& \frac{\partial^{2} F_{2}}{\partial t \partial x}=-\frac{\partial^{2} F_{1}}{\partial x^{2}}=-\frac{\partial^{2} F_{3}}{\partial x \partial z}=\frac{\partial^{2} F_{4}}{\partial y \partial x} \\
& \frac{\partial^{2} F_{2}}{\partial y \partial t}=-\frac{\partial^{2} F_{1}}{\partial y \partial x}=-\frac{\partial^{2} F_{3}}{\partial y \partial z}=\frac{\partial^{2} F_{4}}{\partial y^{2}} \\
& \frac{\partial^{2} F_{2}}{\partial z \partial t}=-\frac{\partial^{2} F_{1}}{\partial z \partial x}=-\frac{\partial^{2} F_{3}}{\partial z^{2}}=\frac{\partial^{2} F_{4}}{\partial z \partial y} .
\end{aligned}
$$

Deriving the conditions of equation (7), we obtain:

$$
\begin{aligned}
& \frac{\partial^{2} F_{3}}{\partial t^{2}}=-\frac{\partial^{2} F_{1}}{\partial t \partial y}=-\frac{\partial^{2} F_{2}}{\partial t \partial z}=\frac{\partial^{2} F_{4}}{\partial t \partial x} \\
& \frac{\partial^{2} F_{3}}{\partial t \partial x}=-\frac{\partial^{2} F_{1}}{\partial x \partial y}=-\frac{\partial^{2} F_{2}}{\partial x \partial z}=\frac{\partial^{2} F_{4}}{\partial x^{2}} \\
& \frac{\partial^{2} F_{3}}{\partial y \partial t}=-\frac{\partial^{2} F_{1}}{\partial y^{2}}=-\frac{\partial^{2} F_{2}}{\partial z \partial y}=\frac{\partial^{2} F_{4}}{\partial y \partial x} \\
& \frac{\partial^{2} F_{3}}{\partial t \partial z}=-\frac{\partial^{2} F_{1}}{\partial z \partial y}=-\frac{\partial^{2} F_{2}}{\partial z^{2}}=\frac{\partial^{2} F_{4}}{\partial z \partial x} .
\end{aligned}
$$

And finally, in deriving the conditions of equation (8), it follows that:

$$
\begin{aligned}
& \frac{\partial^{2} F_{4}}{\partial t^{2}}=\frac{\partial^{2} F_{1}}{\partial t \partial z}=-\frac{\partial^{2} F_{2}}{\partial t \partial y}=-\frac{\partial^{2} F_{3}}{\partial t \partial x} \\
& \frac{\partial^{2} F_{4}}{\partial t \partial x}=\frac{\partial^{2} F_{1}}{\partial x \partial z}=-\frac{\partial^{2} F_{2}}{\partial x \partial y}=-\frac{\partial^{2} F_{3}}{\partial x^{2}} \\
& \frac{\partial^{2} F_{4}}{\partial y \partial t}=\frac{\partial^{2} F_{1}}{\partial y \partial z}=-\frac{\partial^{2} F_{2}}{\partial y^{2}}=-\frac{\partial^{2} F_{3}}{\partial y \partial x} \\
& \frac{\partial^{2} F_{4}}{\partial t \partial z}=\frac{\partial^{2} F_{1}}{\partial z^{2}}=-\frac{\partial^{2} F_{2}}{\partial z \partial y}=-\frac{\partial^{2} F_{3}}{\partial z \partial x} .
\end{aligned}
$$


Correlating groups of partial derivatives in (9), (10), (11) and (12), then immediately follows the Laplace-like Equations:

$$
\begin{aligned}
& \frac{\partial^{2} F_{1}}{\partial^{2} t^{2}}+\frac{\partial^{2} F_{1}}{\partial x^{2}}+\frac{\partial^{2} F_{1}}{\partial y^{2}}+\frac{\partial^{2} F_{1}}{\partial z^{2}}=0 \\
& \frac{\partial^{2} F_{2}}{\partial t^{2}}+\frac{\partial^{2} F_{2}}{\partial x^{2}}+\frac{\partial^{2} F_{2}}{\partial y^{2}}+\frac{\partial^{2} F_{2}}{\partial z^{2}}=0 \\
& \frac{\partial^{2} F_{3}}{\partial t^{2}}+\frac{\partial^{2} F_{3}}{\partial x^{2}}+\frac{\partial^{2} F_{3}}{\partial y^{2}}+\frac{\partial^{2} F_{3}}{\partial z^{2}}=0
\end{aligned}
$$

and

$$
\frac{\partial^{2} F_{4}}{\partial t^{2}}+\frac{\partial^{2} F_{4}}{\partial x^{2}}+\frac{\partial^{2} F_{4}}{\partial y^{2}}+\frac{\partial^{2} F_{4}}{\partial z^{2}}=0
$$

Taking now into account the functions $F_{3}(t, x, y, z)$ and $F_{4}(t, x, y, z)$ at $(13)$ and (14), and making the limit in these equations when $t$ tends to zero, we have that:

$$
\begin{gathered}
\lim _{t \rightarrow 0}\left[\frac{\partial^{2} F_{3}(t, x, y, z)}{\partial t^{2}}+\frac{\partial^{2} F_{3}(t, x, y, z)}{\partial x^{2}}+\frac{\partial^{2} F_{3}(t, x, y, z)}{\partial y^{2}}+\frac{\partial^{2} F_{3}(t, x, y, z)}{\partial z^{2}}\right] \\
=0
\end{gathered}
$$

and

$$
\lim _{t \rightarrow 0}\left[\frac{\partial^{2} F_{4}(t, x, y, z)}{\partial t^{2}}+\frac{\partial^{2} F_{4}(t, x, y, z)}{\partial x^{2}}+\frac{\partial^{2} F_{4}(t, x, y, z)}{\partial y^{2}}+\frac{\partial^{2} F_{4}(t, x, y, z)}{\partial z^{2}}\right]
$$

As already mentioned earlier, the functions $F_{3}$ and $F_{4}$ are of class $C^{2}$ and thus making the limit as $t$ tends to zero, these functions will depend only of $x, y$ and $z$, and will be denoted by $\varphi(x, y, z)$ and $\Phi(x, y, z)$, respectively. Moreover, in the second set of partial derivatives respect to $t$ in the limit as $t$ tends to zero are allowed constants, and now will be made the following identifications:

$$
\lim _{t \rightarrow 0} \frac{\partial^{2} F_{3}(t, x, y, z)}{\partial t^{2}}=\frac{\rho_{f}}{\varepsilon}
$$

and

$$
\lim _{t \rightarrow 0} \frac{\partial^{2} F_{4}(t, x, y, z)}{\partial t^{2}}=4 \pi G \rho
$$


where $\rho_{f}$ is free charge density, $\varepsilon$ is permittivity of the medium. Furthermore, $\rho$ is density and $G$ is gravitational constant. Soon, with the identifications and the limits indicated above, we have the following equations:

$$
\frac{\partial^{2} \varphi(x, y, z)}{\partial x^{2}}+\frac{\partial^{2} \varphi(x, y, z)}{\partial y^{2}}+\frac{\partial^{2} \varphi(x, y, z)}{\partial z^{2}}=-\frac{\rho_{f}}{\varepsilon}
$$

and

$$
\frac{\partial^{2} \Phi(x, y, z)}{\partial x^{2}}+\frac{\partial^{2} \Phi(x, y, z)}{\partial y^{2}}+\frac{\partial^{2} \Phi(x, y, z)}{\partial z^{2}}=-4 \pi G \rho
$$

There is the possibility of determining the solutions of the equations above, but considering that they are related by the Cauchy-Riemann conditions, after the treatment is again considered the limit when $t$ tends to zero. Therefore, a system of partial differential equations, which arise from the Riemann Cauchy like conditions, is presented only for the functions $F_{3}$ and $F_{4}$. It follows that:

$$
\begin{array}{ll}
\frac{\partial^{2} F_{3}}{\partial t \partial y}=\frac{\partial^{2} F_{4}}{\partial t \partial z}, & \frac{\partial^{2} F_{3}}{\partial x \partial y}=\frac{\partial^{2} F_{4}}{\partial x \partial z}, \\
\frac{\partial^{2} F_{3}}{\partial y^{2}}=\frac{\partial^{2} F_{4}}{\partial z \partial y}, & \frac{\partial^{2} F_{3}}{\partial z \partial y}=\frac{\partial^{2} F_{4}}{\partial z^{2}}, \\
-\frac{\partial^{2} F_{3}}{\partial t \partial z}=\frac{\partial^{2} F_{4}}{\partial t \partial y}, & -\frac{\partial^{2} F_{3}}{\partial x \partial z}=\frac{\partial^{2} F_{4}}{\partial y \partial x}, \\
-\frac{\partial^{2} F_{3}}{\partial y \partial z}=\frac{\partial^{2} F_{4}}{\partial y^{2}}, & -\frac{\partial^{2} F_{3}}{\partial z^{2}}=\frac{\partial^{2} F_{4}}{\partial z \partial y}, \\
\frac{\partial^{2} F_{3}}{\partial t^{2}}=\frac{\partial^{2} F_{4}}{\partial t \partial x}, & \frac{\partial^{2} F_{3}}{\partial t \partial x}=\frac{\partial^{2} F_{4}}{\partial x^{2}}, \\
\frac{\partial^{2} F_{3}}{\partial y \partial t}=\frac{\partial^{2} F_{4}}{\partial y \partial x}, & \frac{\partial^{2} F_{3}}{\partial t \partial z}=\frac{\partial^{2} F_{4}}{\partial z \partial x}, \\
\frac{\partial^{2} F_{4}}{\partial t^{2}}=-\frac{\partial^{2} F_{3}}{\partial t \partial x}, & \frac{\partial^{2} F_{4}}{\partial t \partial x}=-\frac{\partial^{2} F_{3}}{\partial x^{2}}, \\
\frac{\partial^{2} F_{4}}{\partial y \partial t}=-\frac{\partial^{2} F_{3}}{\partial y \partial x}, & \frac{\partial^{2} F_{4}}{\partial t \partial z}=-\frac{\partial^{2} F_{3}}{\partial z \partial x},
\end{array}
$$




$$
\begin{array}{ll}
\lim _{t \rightarrow 0} \frac{\partial^{2} F_{4}}{\partial t^{2}}=4 \pi G \rho, & \lim _{t \rightarrow 0} \frac{\partial^{2} F_{3}}{\partial t^{2}}=\frac{\rho_{f}}{\varepsilon}, \\
\frac{\partial F_{3}}{\partial y}=\frac{\partial F_{4}}{\partial z}, & -\frac{\partial F_{3}}{\partial z}=\frac{\partial F_{4}}{\partial y}, \\
\frac{\partial F_{3}}{\partial t}=\frac{\partial F_{4}}{\partial x}, & \frac{\partial F_{4}}{\partial t}=-\frac{\partial F_{3}}{\partial x} .
\end{array}
$$

The above system has the following solution (solution that verifies the Laplace like Equation for $F_{3}$ and $F_{4}$ ), where $C_{1}$ and $C_{2}$ are constants. Hence it follows that:

$$
\begin{array}{r}
F_{3}(t, x, y, z)=-\frac{1}{2}\left(\frac{\rho_{f}}{\varepsilon}\right) x^{2}-\left(4 \pi \rho G t+f_{1}(z-y i)+f_{2}(z+y i)\right) x \\
+\frac{1}{2}\left(\frac{\rho_{f}}{\varepsilon}\right) t^{2}+\left(i f_{1}(z-y i)-i f_{2}(z+y i)+C_{1}\right) t+i f_{3}(z-y i) \\
-i f_{4}(z+y i)+C_{2},
\end{array}
$$

and

$$
\begin{aligned}
F_{4}(t, x, y, z)=\frac{1}{2}(4 \pi \rho G) t^{2}-\left(\left(\frac{\rho_{f}}{\varepsilon}\right) x+f_{1}(z-i y)+f_{2}(z+i y)\right) t & \\
-\frac{1}{2}(4 \pi \rho G) x^{2}+\left(i f_{1}(z-i y)-i f_{2}(z+i y)+C_{1}\right) x & +f_{3}(z-y) \\
& +f_{4}(z+i y) .
\end{aligned}
$$

Making $F_{3}-i F_{4}$ the threshold $t$ tends to zero, ie, $F_{3}(x, y, z)-i F_{4}(x, y, z)$ we have that:

$$
\begin{array}{r}
F_{3}(x, y, z)-i F_{4}(x, y, z)=-\frac{1}{2}\left(\frac{\rho_{f}}{\varepsilon}\right) x^{2}+\frac{1}{2}(4 \pi G \rho) x^{2} i-2 f_{2}(z+i y) x \\
+C_{2}-C_{1} x i-2 i f_{4}(z+i y) .
\end{array}
$$

On the other hand, performing the partial derivatives of the above functions and taken to the limit when $t \rightarrow 0$, and making the appropriate identifications, we have that:

$$
\begin{aligned}
& i \frac{\partial F_{3}}{\partial y}(x, y, z)=-D f_{1}(z-i y) x+D f_{2}(z+i y) x+i D f_{3}(z-i y)+i D f_{4}(z+i y), \\
& \frac{\partial F_{4}}{\partial y}(x, y, z)=D f_{1}(z-i y) x+D f_{2}(z+i y) x-i D f_{3}(z-i y)+i D f_{4}(z+i y),
\end{aligned}
$$


which together give us:

$$
\frac{\partial F_{4}}{\partial y}(x, y, z)+i \frac{\partial F_{3}}{\partial y}(x, y, z)=2 D f_{2}(z+i y) x+2 i D f_{4}(z+i y) x .
$$

Similarly,

$$
\begin{aligned}
& i \frac{\partial F_{3}}{\partial z}(x, y, z)=-i D f_{1}(z-i y) x-i D f_{2}(z+i y) x-D f_{3}(z-i y)+D f_{4}(z+i y), \\
& \frac{\partial F_{4}}{\partial z}(x, y, z)=i D f_{1}(z-i y) x-i D f_{2}(z+i y) x+D f_{3}(z-i y)+i D f_{4}(z+i y),
\end{aligned}
$$

which together generate the following equality:

$$
\frac{\partial F_{4}}{\partial z}(x, y, z)+i \frac{\partial F_{3}}{\partial z}(x, y, z)=-2 i D f_{2}(z+i y) x+2 D f_{4}(z+i y)
$$

Finally, by taking the sum:

$$
\begin{aligned}
\frac{\partial F_{4}}{\partial y}(x, y, z)+i \frac{\partial F_{3}}{\partial y}(x, y, z)-\frac{\partial F_{3}}{\partial z}(x, y, z)+i \frac{\partial F_{4}}{\partial z}(x, y, z) & \\
& =4 D f_{1}(z+i y) x+4 i D f_{4}(z+i y)
\end{aligned}
$$

or

$$
\begin{array}{r}
\left(\frac{\partial F_{4}}{\partial y}(x, y, z)-\frac{\partial F_{3}}{\partial z}(x, y, z)\right)+i\left(\frac{\partial F_{3}}{\partial y}(x, y, z)+\frac{\partial F_{4}}{\partial z}(x, y, z)\right) \\
=4 D f_{1}(z+i y) x+4 i D f_{4}(z+i y) .
\end{array}
$$

Integrating the terms of $f_{1}(z+i y)$ and $f_{4}(z+i y)$ we have:

$$
4 D f_{1}(z+i y) x=\left(\frac{\partial F_{4}}{\partial y}(x, y, z)-\frac{\partial F_{3}}{\partial z}(x, y, z)\right)
$$

that is equal to

$$
f_{1}(z+i y) x=-\frac{1}{2}\left(F_{3}(x, y, z)-F_{3}\left(x, y, z_{0}\right)\right)+\frac{i}{2}\left(F_{4}(x, y, z)-F_{4}\left(x, y_{0}, z\right)\right) \text {. }
$$

Similarly, we have:

$$
f_{4}(z+i y)=\frac{1}{2}\left(F_{4}(x, y, z)-F_{4}\left(x, y, z_{0}\right)\right)+\frac{i}{2}\left(F_{3}(x, y, z)-F_{3}\left(x, y_{0}, z\right)\right) .
$$

Substituting the above results integrated $y_{0}$ to $y$ and $z_{0}$ by $z$ and substituting in equation (24) it follows that: 


$$
\begin{aligned}
(-1)\left[F_{3}(x, y, z)-F_{3}\left(x, y, z_{0}\right)-\right. & \left.F_{3}\left(x, y_{0}, z\right)\right] \\
& +i\left[F_{4}(x, y, z)-F_{4}\left(x, y, z_{0}\right)-F_{4}\left(x, y_{0}, z\right)\right] \\
=\left(-\frac{1}{2}\left(\frac{\rho_{f}}{\varepsilon}\right) x^{2}+\right. & \left.C_{2}\right)+i\left(\frac{1}{2}(4 \pi G \rho) x^{2}+C_{1} x\right)
\end{aligned}
$$

or

$$
\begin{aligned}
{\left[F_{3}(x, y, z)-F_{3}\left(x, y, z_{0}\right)\right.} & \left.-F_{3}\left(x, y_{0}, z\right)\right]^{2}+\left[F_{4}(x, y, z)-F_{4}\left(x, y, z_{0}\right)-F_{4}\left(x, y_{0}, z\right)\right]^{2} \\
& =\left(\frac{1}{2}\left(\frac{\rho_{f}}{\varepsilon}\right) x^{2}-C_{2}\right)^{2}+\left(\frac{1}{2}(4 \pi G \rho) x^{2}+C_{1} x\right)^{2} \cdot
\end{aligned}
$$

The results of this work can be summarized in the following theorem:

Theorem 2. Let $f(q)$ quaternionic function that satisfies the CauchyRiemann conditions. If $f(q)$ is of class $C^{2}$, then it is possible to determine a relationship between gravitational and electrical potential as listed below:

$$
\begin{aligned}
{\left[F_{3}(x, y, z)-F_{3}\left(x, y, z_{0}\right)\right.} & \left.-F_{3}\left(x, y_{0}, z\right)\right]^{2}+\left[F_{4}(x, y, z)-F_{4}\left(x, y, z_{0}\right)-F_{4}\left(x, y_{0}, z\right)\right]^{2} \\
& =\left(\frac{1}{2}\left(\frac{\rho_{f}}{\varepsilon}\right) x^{2}-C_{2}\right)^{2}+\left(\frac{1}{2}(4 \pi G \rho) x^{2}+C_{1} x\right)^{2} .
\end{aligned}
$$

\section{Conclusion}

The present results in the previous sections, showed the feasibility of obtaining the equations of Laplace through the Cauchy-Riemann like conditions for quaternions. This fact will allow the relationship between equations that can explain such physical phenomena e. g. the possibility of a geometrical coupling regarding gravitational and electric fields. You can also use the above equations as a way of stating the theorem for harmonic functions that satisfy the Cauchy conditions.

\section{References}

[1] J.M. Machado, M.F. Borges, New ramrks on the differenciability of hypercomplex functions, International Journal of Pure and Applied Mathematics, 8, No. 1 (2002), 85-101.

[2] J. Marão, M.F. Borges, A note on the hypercomplex Riemann-Cauchy like relations for quaternions and laplace equations, In: notes. 